\title{
Review of: "The impact of housing conditions on porcine mesenchymal stromal/stem cell populations differ between adipose tissue and skeletal muscle"
}

\author{
Suchitra Gopinath
}

Potential competing interests: The author(s) declared that no potential competing interests exist.

The following preprint is a descriptive study reporting proportions of multipotent mesenchymal/stromal cells defined by specific marker status in skeletal muscle and adipose tissue of pigs that have been housed in different hygienic conditions and also differ in their feed efficiency (RFI). From earlier studies, several combinations of surface markers have been used to isolate MSCs from these two specific tissues. In the context of adipose tissue MSCs, it is likely that the isolated populations within the stroma vascular fraction (SVF) or within cell suspensions from muscle tissue various studies represent fibroblasts, vascular smooth muscle cells, and progenitors with differing differentiation potential. Therefore, albeit a minor point, titles such as "Muscle cell Isolation" or "Adipose Cell Isolation" in the Methods section would be more accurately termed as "Isolation of mononuclear cells from Muscle and Adipose tissue" respectively. Demonstration of lineage potential in such populations and/or comparisons of differentiation potential of MSCs with existing surface markers therefore would be useful placeholders in the context of animal production and altering lean muscle to fat ratios, and impart significance to these descriptive findings. In the current paper, a combination of CD45, CD56, and CD34 appear to be the dominant fraction in adipose tissue MSCs. Other studies have shown that most of the cells in the SVF that adhered to the culture plate were $\mathrm{CD} 31^{-} / \mathrm{CD} 4^{+} / \mathrm{CD} 45^{-} / \mathrm{CD} 146^{-}$and that multilineage potential was inherent in the CD31 negative fraction. (Boquest et al, 2005). On the contrary, the presence of CD146 enhanced myogenic potential from muscle-derived MSCs (Sacchetti et al, 2016). A further advantage of exploring already established markers for MSCs isolated from muscle and adipose tissue, is these populations have been demonstrated to possess specific tissue differentiation potential. Thus, the significance of proportional differences in the resident MSCs in different tissues can be compared for tissue differentiation potentials amongst the low and high RFI groups and hygienic conditions. For instance, do the CD45-CD56-CD34- population in adipose tissue that is increased in poor housing conditions compared to the CD45-CD56+CD34- population display comparable differences in adipogenic potentials in culture? How does the same population, CD45-CD56CD34- exhibit differential susceptibility in adipose tissue and muscle tissue (increased in fat and decreased in muscle) in response to the same cue, i.e., poor hygiene? While the authors have previously demonstrated myogenic potentials of MSC populations with limited markers in previous studies as stated in their discussion, it would be pertinent to test the current populations of MSCs as defined by an expanded set of markers in both tissues in terms of differentiation potentials and observe comparable 
differences between the housing groups. This is especially relevant since cell populations isolated using several surface markers might be a subset of cell populations isolated using a couple of surface markers. Another observation from the differential analysis reveals that CD56+ presence in adipose tissue MSCs increases their susceptibility to poor hygienic conditions. CD56 null MSCs have been demonstrated to display a marked decrease in adipogenic potentials (Yang et al, 2011). Thus, it would be predicted that the decrease in CD45-CD56+CD34+ might result in animals with reduced backfat and increased loin/backfat ratios. Similarly, it is the differential CD34 expression status in muscle that is an indication of susceptibility to poor housing conditions suggesting a link between increased CD34 expression and inflammation in muscle MSCs resulting in reduced lean muscle in these animals. Overall, while the authors have chosen to limit their findings to descriptive observations, coupling in vitro experiments with these studies would enhance the significance of the findings, in addition to raising important questions on multilineage potential of tissue stem cells. 